\title{
Auto-Bäcklund Transformation and Extended Tanh-Function Methods to Solve the Time-Dependent Coefficients Calogero-Degasperis Equation
}

\author{
Rehab M. El-Shiekh \\ Department of Mathematics, Faculty of Education, Ain Shams University, Cairo, Egypt \\ Email: rehab el shiekh@yahoo.com
}

Received 17 May 2015; accepted 27 June 2015; published 30 June 2015

Copyright (C) 2015 by author and Scientific Research Publishing Inc. This work is licensed under the Creative Commons Attribution International License (CC BY). http://creativecommons.org/licenses/by/4.0/

(c) (i) Open Access

\begin{abstract}
In this paper, the Auto-Bäcklund transformation connected with the homogeneous balance method (HB) and the extended tanh-function method are used to construct new exact solutions for the time-dependent coefficients Calogero-Degasperis (VCCD) equation. New soliton and periodic solutions of many types are obtained. Furthermore, the soliton propagation is discussed under the effect of the variable coefficients.
\end{abstract}

\section{Keywords}

Auto-Bäcklund Transformation, Homogeneous Balance Method, The Extended Tanh-Function Method, The Time-Dependent Coefficients Calogero-Degasperis Equation, Exact Solutions

\section{Introduction}

Recently, investigation of exact solutions for nonlinear partial differential equations (NPDEs) with variable coefficients plays an important role in modern nonlinear science because NPDEs with variable coefficients reflect the real thing even more than those with constant.

One of the most important NPDEs is the time-dependent coefficients Calogero-Degasperis (VCCD) equation [1]

$$
u_{x t}+\alpha(t) u_{x} u_{x y}+\beta(t) u_{y} u_{x x}+\gamma(t) u_{x x x y}=0
$$

How to cite this paper: El-Shiekh, R.M. (2015) Auto-Bäcklund Transformation and Extended Tanh-Function Methods to Solve the Time-Dependent Coefficients Calogero-Degasperis Equation. American Journal of Computational Mathematics, 5, 215-223. http://dx.doi.org/10.4236/ajcm.2015.52018 
where $\alpha(t), \beta(t)$ and $\gamma(t)$ are arbitrary functions. The VCCD equation describes the $(2+1)$-dimensional interaction of the Riemann wave propagating along the $\mathrm{y}$-axis with a long wave along the $\mathrm{x}$-axis. Many exact solutions have been found for Equation (1) by using symmetry method [1]. Equation (1) with $\alpha, \beta$ and $\gamma$ as constants was first constructed by Bogoyavlenskii and Schiff in different ways [2]-[4] and called the CalogeroBogoyavlenskii-Schiff (CBS) equation. Bogoyavlenskii used the modified Lax formalism, whereas Schiff derived the same equation by reducing the self-dual Yang-Mills equation. The CBS equation has been solved by using Hirota's bilinear method [5] and symmetry method [6].

The objective of this paper is to apply the auto-Bäcklund transformation method and the extended tanhfunction method on the VCCD equation, to find more general new solitonic and periodic exact solutions.

\section{Auto-Bäcklund Transformation}

We can obtain Auto-Bäcklund transformation by using HB method [7]-[10] as follows.

Step 1: We consider the exact solution of (1) in the form

$$
u=\frac{\partial^{n}}{\partial x^{n}} f(w)+v,
$$

where $f=f(w)$ and $w=w(t, x, y)$ are undetermined functions, $v=v(t, x, y)$ is a solution of (1).

According to the HB method $n$ can be determined by balancing the linear term of the highest order derivative and the highest nonlinear term of $u$ in (1).

Therefore, $n=1$ and

$$
u(t, x, y)=\frac{\partial}{\partial x} f+v
$$

Substituting (3) into (1), we get

$$
\begin{aligned}
& {\left[(\alpha(t)+\beta(t)) f^{\prime \prime} f^{\prime \prime \prime}+\gamma(t) f^{\prime \prime \prime \prime \prime}\right] w_{x}^{4} w_{y}+\left[\alpha(t)\left(2 w_{x}^{3} w_{x y}+w_{x}^{2} w_{y} w_{x x}\right)+3 \beta(t) w_{x}^{2} w_{y} w_{x x}\right]\left(f^{\prime \prime}\right)^{2}} \\
& +\left[\alpha(t) w_{x}^{2} w_{y} w_{x x}+\beta(t) w_{x}^{3} w_{x y}\right] f^{\prime \prime \prime}+\left[4 w_{x}^{3} w_{x y}+6 w_{x}^{2} w_{x x} w_{y}\right] \gamma(t) f^{\prime \prime \prime \prime} \\
& +\left[\alpha(t)\left(w_{x}^{2} w_{x x y}+2 w_{x} w_{x x} w_{x y}+w_{y} w_{x x}^{2}\right)+\beta(t)\left(3 w_{x} w_{x x} w_{x y}+w_{x} w_{y} w_{x x x}\right)\right] f^{\prime \prime} \\
& +\left[\alpha(t) w_{x x} w_{x x y}+\beta(t) w_{x y} w_{x x x}\right]\left(f^{\prime}\right)^{2}+\left[w_{t} w_{x}^{2}+\alpha(t) v_{x} w_{y} w_{x}^{2}+\beta(t) v_{y} w_{x}^{3}\right. \\
& \left.+\gamma(t)\left(12 w_{x} w_{x x} w_{x y}+6 w_{x}^{2} w_{x x y}+3 w_{y} w_{x x}^{2}+4 w_{x} w_{y} w_{x x x}\right)\right] f^{\prime \prime \prime} \\
& +\left[2 w_{x} w_{x x}+w_{t} w_{x x}+\alpha(t)\left(w_{x}^{2} v_{x y}+2 w_{x} w_{x y} v_{x}+w_{y} w_{x x} v_{x}\right)\right. \\
& \left.+\beta(t)\left(3 w_{x} w_{x x} v_{y}+v_{x x x} w_{x} w_{y}\right)+\gamma(t)\left(4 w_{x y} w_{x x x}+6 w_{x x} w_{x x y}+4 w_{x} w_{x x x y}+w_{y} w_{x x x x}\right)\right] f^{\prime \prime} \\
& +\left[w_{x x t}+\alpha(t)\left(w_{x x} v_{x y}+w_{x x y} v_{x}\right)+\beta(t)\left(w_{x x x} v_{y}+v_{x x} w_{x y}\right)+\gamma(t) w_{x x x x y}\right] f^{\prime} \\
& +v_{x t}+\alpha(t) v_{x} v_{x y}+\beta(t) v_{x x} v_{y}+\gamma(t) v_{x x x y}=0
\end{aligned}
$$

Step 2: To make (4) as a linear equation in $f$ we assume that,

$$
f(w)=c \ln w, \quad \text { where } c \text { is constant }
$$

So that, we have the following relations

$$
f^{\prime \prime} f^{\prime \prime \prime}=-\frac{c}{12} f^{\prime \prime \prime \prime \prime},\left(f^{\prime \prime}\right)^{2}=-\frac{c}{6} f^{\prime \prime \prime \prime}, f f^{\prime \prime \prime}=-\frac{c}{3} f^{\prime \prime \prime \prime}, f^{f^{\prime \prime}}=-\frac{c}{2} f^{\prime \prime \prime}, f^{\prime 2}=-c f^{\prime \prime}
$$

Substitute from relations (6) into (4) and equating the linear coefficients $f^{\prime \prime \prime \prime}, f^{\prime \prime \prime \prime}, \cdots, f^{\prime}$ by zero, the following partial differential system is obtained

$$
\left[-\frac{c}{12}(\alpha(t)+\beta(t))+\gamma(t)\right] w_{x}^{4} w_{y}=0,
$$




$$
\begin{gathered}
{\left[4 w_{x}^{3} w_{x y}+6 w_{x}^{2} w_{x x} w_{y}\right] \gamma(t)-\frac{c}{6}\left[\alpha(t)\left(2 w_{x}^{3} w_{x y}+w_{x}^{2} w_{y} w_{x x}\right)+3 \beta(t) w_{x}^{2} w_{y} w_{x x}\right]} \\
-\frac{c}{3}\left[\alpha(t) w_{x}^{2} w_{y} w_{x x}+\beta(t) w_{x}^{3} w_{x y}\right]=0, \\
w_{t} w_{x}^{2}+\alpha(t) v_{x} w_{y} w_{x}^{2}+\beta(t) v_{y} w_{x}^{3}+\gamma(t)\left(12 w_{x} w_{x x} w_{x y}+6 w_{x}^{2} w_{x x y}+3 w_{y} w_{x x}^{2}+4 w_{x} w_{y} w_{x x x}\right) \\
-\frac{c}{2}\left[\alpha(t)\left(w_{x}^{2} w_{x x y}+2 w_{x} w_{x x} w_{x y}+w_{y} w_{x x}^{2}\right)+\beta(t)\left(3 w_{x} w_{x x} w_{x y}+w_{x} w_{y} w_{x x x}\right)\right]=0, \\
2 w_{x} w_{x t}+w_{t} w_{x x}+\alpha(t)\left(w_{x}^{2} v_{x y}+2 w_{x} w_{x y} v_{x}+w_{y} w_{x x} v_{x}\right)+\beta(t)\left(3 w_{x} w_{x x} v_{y}+v_{x x x} w_{x} w_{y}\right) \\
+\gamma(t)\left(4 w_{x y} w_{x x x}+6 w_{x x} w_{x x y}+4 w_{x} w_{x x x y}+w_{y} w_{x x x x}\right)-c\left[\alpha(t) w_{x x} w_{x x y}+\beta(t) w_{x y} w_{x x x}\right]=0, \\
w_{x x t}+\alpha(t)\left(w_{x x} v_{x y}+w_{x x y} v_{x}\right)+\beta(t)\left(w_{x x x} v_{y}+v_{x x} w_{x y}\right)+\gamma(t) w_{x x x x y}=0, \\
v_{x t}+\alpha(t) v_{x} v_{x y}+\beta(t) v_{x x} v_{y}+\gamma(t) v_{x x x y}=0 .
\end{gathered}
$$

Step 3: To solve the previous system, assume that

$$
w=C+\exp (k x+r y-\theta(t)),
$$

where $C, k, r$ are arbitrary constants and $\theta(t)$ is an arbitrary function of $t$. Then, we obtain the following relations

$$
\begin{gathered}
\gamma(t)=\frac{c}{12}(\alpha(t)+\beta(t)) \\
\theta(t)=\frac{c k^{2} r}{12} \int(\alpha(t)+\beta(t)) \mathrm{d} t, \quad v=v_{0} \text { is a constant }
\end{gathered}
$$

By substitution from (8-10) into (3) using (5), we obtain the following one-soliton solution for the VCCD equation under condition (9)

$$
u_{1}(x, y, t)=\frac{k c \exp \left(k x+r y-\frac{c k^{2} r}{12} \int(\alpha(t)+\beta(t)) \mathrm{d} t\right)}{C+\exp \left(k x+r y-\frac{c k^{2} r}{12} \int(\alpha(t)+\beta(t)) \mathrm{d} t\right)}+v_{0}
$$

By using the following two useful formulas [11]

$$
\begin{gathered}
\frac{\exp (\zeta)}{C+\exp (\zeta)}= \begin{cases}1 & \text { for } C=0 \\
\frac{1}{2}\left[\tanh \frac{1}{2}(\zeta-\ln C)+1\right] & \text { for } C>0 \\
\frac{1}{2}\left[\operatorname{coth} \frac{1}{2}(\zeta-\ln (-C))+1\right] & \text { for } C<0\end{cases} \\
\frac{\exp i(\zeta)}{C+\exp i(\zeta)}= \begin{cases}1 & \text { for } C=0 \\
\frac{1}{2}\left[i \tan \frac{1}{2}(\zeta+i \ln (C))+1\right] & \text { for } C>0 \\
\frac{1}{2}\left[-i \cot \frac{1}{2}(\zeta+i \ln (-C))+1\right] & \text { for } C<0\end{cases}
\end{gathered}
$$

We obtain the following kink-type soliton and periodic solutions respectively 


$$
\begin{aligned}
& u_{2}=\frac{k c}{2}\left[\tanh \left[\frac{1}{2}\left(k x+r y-\frac{c k^{2} r}{12} \int(\alpha(t)+\beta(t)) \mathrm{d} t-\ln (C)\right)\right]+1\right]+v_{0} \\
& u_{3}=\frac{k c}{2}\left[\operatorname{coth}\left[\frac{1}{2}\left(k x+r y-\frac{c k^{2} r}{12} \int(\alpha(t)+\beta(t)) \mathrm{d} t+\ln (-C)\right)\right]+1\right]+v_{0}
\end{aligned}
$$

Analogously, we assume that $k=i k_{1}$ and $r=i r_{1}$ in (11), where $k_{1}$ and $r_{1}$ are real constants. So the following new periodic solutions for the VCCD equation are obtained

$$
\begin{gathered}
u_{4}=\frac{-k_{1} C}{2}\left[\tan \left[\frac{1}{2}\left(k_{1} x+r_{1} y+\frac{c k_{1}^{2} r_{1}}{12} \int(\alpha(t)+\beta(t)) \mathrm{d} t+i \ln (C)\right)\right]+1\right]+v_{0} \\
u_{5}=\frac{k_{1} C}{2}\left[\cot \left[\frac{1}{2}\left(k_{1} x+r_{1} y+\frac{c k_{1}^{2} r}{12} \int(\alpha(t)+\beta(t)) \mathrm{d} t+i \ln (-C)\right)\right]+1\right]+v_{0}
\end{gathered}
$$

\section{The Extended Tanh-Function Method}

In this section, we are going to find more new exact solutions for the VCCD equation using direct integration and extended tanh-function method [12] [13]. Assume that

$$
u(x, y, t)=g(\xi) \text {, where } \xi=k_{2} x+r_{2} y-\lambda(t),
$$

where $k_{2}$ and $r_{2}$ are arbitrary constants and $\lambda(t)$ is an arbitrary function of $t$.

By substitution in (1), we have

$$
-k_{2} \lambda^{\prime}(t) g^{\prime \prime}+k_{2}^{2} r_{2}(\alpha(t)+\beta(t)) g^{\prime} g^{\prime \prime}+k_{2}^{3} r_{2} \gamma(t) g^{\prime \prime \prime \prime}=0
$$

To make the previous Equation (19) be an ordinary differential equation, we have found

$$
\begin{gathered}
\lambda(t)=\int(\alpha(t)+\beta(t)) \mathrm{d} t \\
\gamma(t)=\frac{a}{k_{2}^{2} r_{2}}(\alpha(t)+\beta(t)), \text { where } a \text { is an arbitrary constant. }
\end{gathered}
$$

Therefore, (19) becomes

$$
-g^{\prime \prime}+k_{2} r_{2} g^{\prime} g^{\prime \prime}+a g^{\prime \prime \prime \prime}=0
$$

By Integrating (22) twice, we get

$$
a g^{\prime \prime 2}+\frac{k_{2} r_{2}}{3} g^{\prime 3}-g^{\prime 2}=c_{1} g^{\prime}+c_{2},
$$

where $c_{1}$ and $c_{2}$ are integration constants. Assume that $R=g^{\prime}$, then (23) becomes

$$
R^{\prime 2}=\frac{1}{a}\left(-\frac{k_{2} r_{2}}{3} R^{3}+R^{2}+c_{1} R+c_{2}\right) .
$$

Now, we apply the extended tanh function method used in [14] to obtain exact travelling wave solutions of Equation (24). Let us assume that Equation (24) has a solution in the form

$$
R(\zeta)=A_{0}+\sum_{i=1}^{N} A_{i} \phi^{i}
$$

where $\phi(\zeta)$ is a solution of the following Riccati equation

$$
\phi^{\prime}=b+\phi^{2}
$$

This Riccati equation has the following solutions 


$$
\begin{array}{cc}
\phi(\zeta)=-\sqrt{-b} \tanh (\sqrt{-b} \zeta), & b<0, \\
\phi(\zeta)=-\sqrt{-b} \operatorname{coth}(\sqrt{-b} \zeta), & b<0, \\
\phi(\zeta)=\sqrt{b} \tan (\sqrt{b} \zeta), & b>0, \\
\phi(\zeta)=-\sqrt{b} \cot (\sqrt{b} \zeta), & b>0, \\
\phi(\zeta)=-\frac{1}{\zeta}, \quad b=0, &
\end{array}
$$

Substitute from (25) into Equation (24) and balance the term $R^{\prime 2}$ with the greatest nonlinear term $R^{3}$, we get that

$$
N=2
$$

Therefore,

$$
R(\zeta)=A_{0}+A_{1} \phi+A_{2} \phi^{2},
$$

where $A_{0}, A_{1}$ and $A_{2}$ are constants to be determined. Then, by substitution from (29) and (26) in (24), and equating the coefficients of $\phi(\zeta)$ and all its powers with zero, we obtain an algebraic system by solving it with mathematica program many values of $A_{0}, A_{1}$ and $A_{2}$ are obtained. We have chosen one of them for simplicity

$$
\begin{aligned}
& A_{0}=\frac{1}{k_{2} r_{2}}\left(1+2 \sqrt{1+k_{2} r_{2} c_{1}}\right), A_{1}=0, A_{2}=\frac{3 \sqrt{1+k_{2} r_{2} c_{1}}}{k_{2} r_{2}}, a=\frac{-1}{4 b} \sqrt{1+k_{2} r_{2} c_{1}}, \\
& C_{2}=\frac{1}{3}\left(\frac{-2}{k_{2}^{2} r_{2}^{2}}-\frac{3 c_{1}}{k_{2} r_{2}}+\frac{2\left(1+k_{2} r_{2} c_{1}\right)^{\frac{3}{2}}}{k_{2}^{2} r_{2}^{2}}\right)
\end{aligned}
$$

By substitution from (30) and (27) in (29), we have got the following exact solutions for Equation (24)

$$
\begin{aligned}
& \left.\begin{array}{l}
R_{1}(\xi)=\frac{1}{k_{2} r_{2}}\left(1+2 \sqrt{1+k_{2} r_{2} c_{1}}\right)-\frac{3 \sqrt{1+k_{2} r_{2} c_{1}}}{k_{2} r_{2}} \tanh ^{2}(\xi), \\
R_{2}(\xi)=\frac{1}{k_{2} r_{2}}\left(1+2 \sqrt{1+k_{2} r_{2} c_{1}}\right)-\frac{3 \sqrt{1+k_{2} r_{2} c_{1}}}{k_{2} r_{2}} \operatorname{coth}^{2}(\xi),
\end{array}\right\}, \text { where } a=\frac{1}{4} \sqrt{1+k_{2} r_{2} c_{1}}, b=-1 \\
& \left.\begin{array}{l}
R_{3}(\xi)=\frac{1}{k_{2} r_{2}}\left(1+2 \sqrt{1+k_{2} r_{2} c_{1}}\right)+\frac{3 \sqrt{1+k_{2} r_{2} c_{1}}}{k_{2} r_{2}} \tan ^{2}(\xi), \\
R_{4}(\xi)=\frac{1}{k_{2} r_{2}}\left(1+2 \sqrt{1+k_{2} r_{2} c_{1}}\right)+\frac{3 \sqrt{1+k_{2} r_{2} c_{1}}}{k_{2} r_{2}} \cot ^{2}(\xi),
\end{array}\right\}, \quad \text { where } a=\frac{-1}{4} \sqrt{1+k_{2} r_{2} c_{1}}, b=1
\end{aligned}
$$

and $c_{2}=\frac{1}{3}\left(\frac{-2}{k_{2}^{2} r_{2}^{2}}-\frac{3 c_{1}}{k_{2} r_{2}}+\frac{2\left(1+k_{2} r_{2} c_{1}\right)^{\frac{3}{2}}}{k_{2}^{2} r_{2}^{2}}\right)$ for Equations (31). By back substitution from (31) into (18) using the relation $R=g^{\prime}$ and (20-21), we have got the following new exact solutions for the VCCD equation.

$$
\begin{aligned}
u_{6}= & \frac{3 \sqrt{1+k_{2} r_{2} c_{1}}}{k_{2} r_{2}} \tanh \left(k_{2} x+r_{2} y-\int(\alpha(t)+\beta(t)) \mathrm{d} t\right) \\
& +\frac{\left(1-\sqrt{1+k_{2} r_{2} c_{1}}\right)}{k_{2} r_{2}}\left(k_{2} x+r_{2} y-\int(\alpha(t)+\beta(t)) \mathrm{d} t\right),
\end{aligned}
$$




$$
\begin{aligned}
u_{7}= & \frac{3 \sqrt{1+k_{2} r_{2} c_{1}}}{k_{2} r_{2}} \operatorname{coth}\left(k_{2} x+r_{2} y-\int(\alpha(t)+\beta(t)) \mathrm{d} t\right) \\
& +\frac{\left(1-\sqrt{1+k_{2} r_{2} c_{1}}\right)}{k_{2} r_{2}}\left(k_{2} x+r_{2} y-\int(\alpha(t)+\beta(t)) \mathrm{d} t\right),
\end{aligned}
$$

where $\gamma(t)=\frac{\sqrt{1+k_{2} r_{2} c_{1}}}{4 k_{2}^{2} r_{2}}(\alpha(t)+\beta(t))$,

$$
\begin{aligned}
u_{8}= & \frac{3 \sqrt{1+k_{2} r_{2} c_{1}}}{k_{2} r_{2}} \tan \left(k_{2} x+r_{2} y-\int(\alpha(t)+\beta(t)) \mathrm{d} t\right) \\
& +\frac{\left(1-\sqrt{1+k_{2} r_{2} c_{1}}\right)}{k_{2} r_{2}}\left(k_{2} x+r_{2} y-\int(\alpha(t)+\beta(t)) \mathrm{d} t\right), \\
u_{9}= & \frac{-3 \sqrt{1+k_{2} r_{2} c_{1}}}{k_{2} r_{2}} \cot \left(k_{2} x+r_{2} y-\int(\alpha(t)+\beta(t)) \mathrm{d} t\right) \\
& +\frac{\left(1-\sqrt{1+k_{2} r_{2} c_{1}}\right)}{k_{2} r_{2}}\left(k_{2} x+r_{2} y-\int(\alpha(t)+\beta(t)) \mathrm{d} t\right),
\end{aligned}
$$

where $\gamma(t)=\frac{-\sqrt{1+k_{2} r_{2} c_{1}}}{4 k_{2}^{2} r_{2}}(\alpha(t)+\beta(t))$.

The following part of this section is devoted to analyzing the influences of the variable coefficients on the solitonic propagation. From the expression of $u_{1}$, we can get the characteristic line of the soliton solution [15]-[17] as

$$
k x+r y-\frac{c k^{2} r}{12} \int(\alpha(t)+\beta(t)) \mathrm{d} t=0
$$

from the previous equation, we have found that there are three arbitrary constants $k, r$ and $c$ so that it is important to control the solitonic velocity in the profile at $y=0$ (or $y$ is constant) by choosing appropriate parameters. Correspondingly, the velocity $v$ of the solitary wave along the $x$-axis can be expressed as

$$
v=\frac{c k r}{12}(\alpha(t)+\beta(t))
$$

Therefore, the propagation direction of the soliton is decided by the sign of $v$ and the solitonic velocity depend on the variables $\alpha(t)$ and $\beta(t)$ and the same can be done for the kink-soliton solution $u_{6}$

The previous figures indicate that how the variable coefficients $\alpha(t), \beta(t)$ affect the evolution of the soliton in Figures 1-6. In Figure 2, Figure 3, Figure 5 and Figure 6, we can see that the solitonic propagation

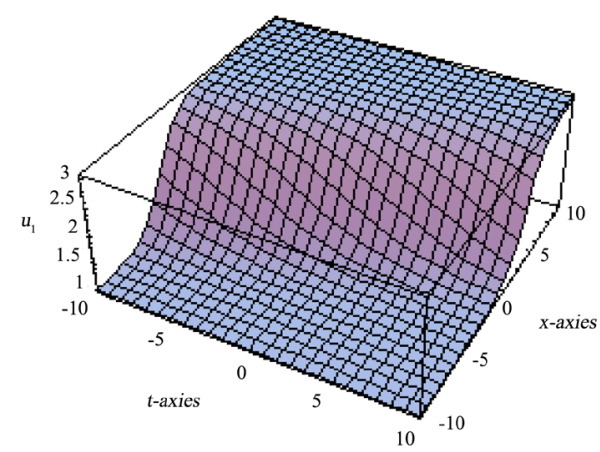

Figure 1. The soliton solution $u_{1}$ with $\alpha(t)=\beta(t)=1$ and $k=r=$ $c=C=v_{0}=1$. 


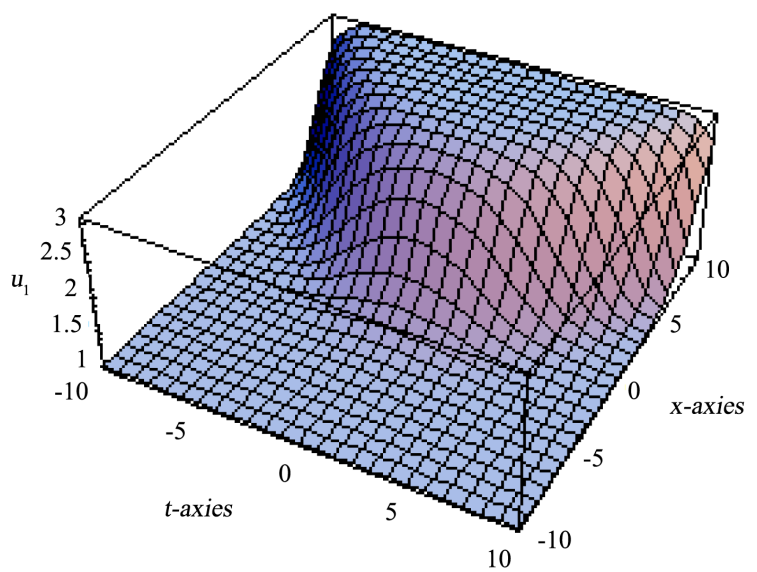

Figure 2. The soliton solution $u_{1}$ with $\alpha(t)=\beta(t)=t$ and $k=r=$ $c=C=v_{0}=1$.

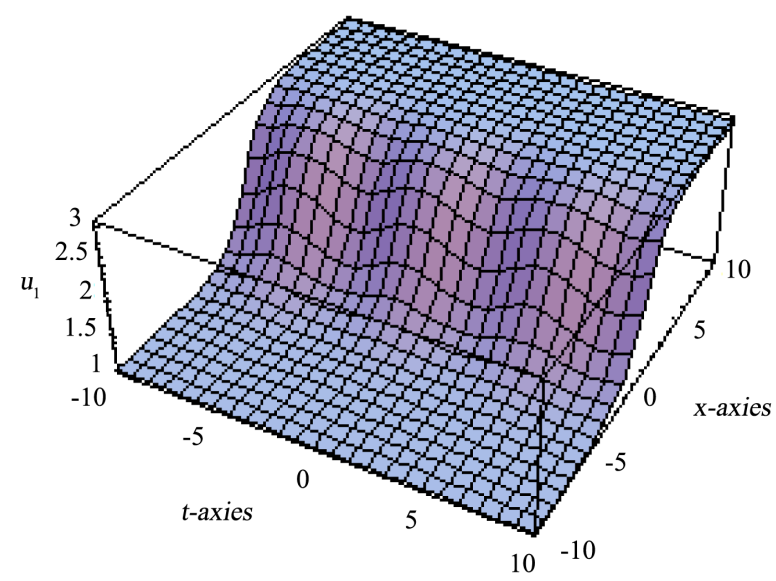

Figure 3. The soliton solution $u_{1}$ with $\alpha(t)=\sin (t), \beta(t)=\cos (t)$ and $k=r=c=C=v_{0}=1$.

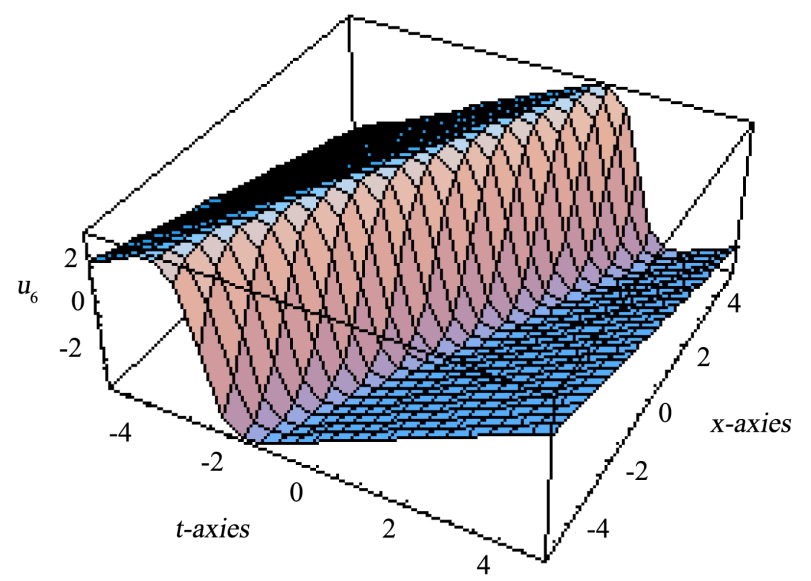

Figure 4. The kink-soliton solution $u_{6}$ with $\alpha(t)=\beta(t)=1$ and $k_{2}=r_{2}=c_{1}=1$.

trajectory is not a straight line anymore. It exhibits as a parabolic and periodic-type propagation respectively. 


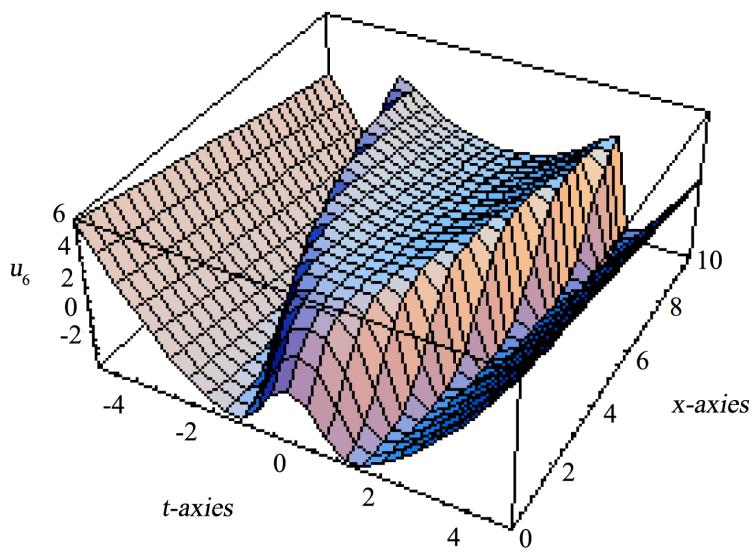

Figure 5. The kink-soliton solution $u_{6}$ with $\alpha(t)=\beta(t)=t$ and $k_{2}=r_{2}=c_{1}=1$.

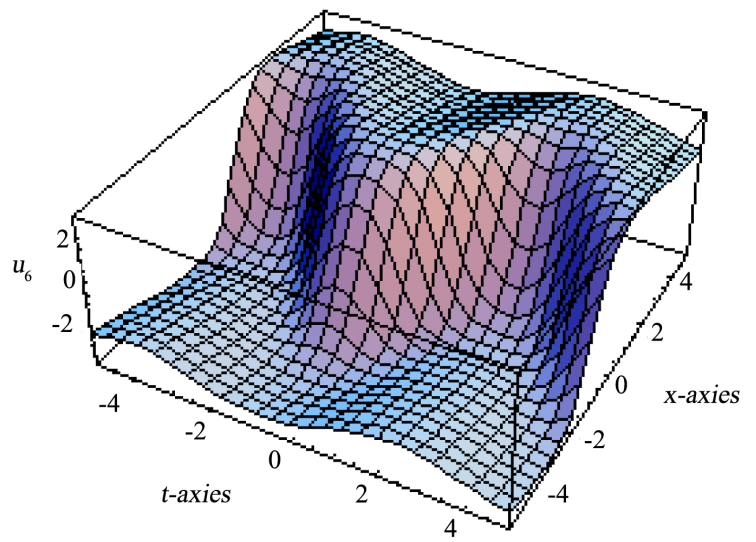

Figure 6. The kink-soliton solution $u_{6}$ with $\alpha(t)=\sin (t), \beta(t)=$ $\cos (t)$ and $k_{2}=r_{2}=c_{1}=1$.

\section{Conclusions}

By using the HB method, we have obtained Auto-Bäcklund transformation and new exact solitary and periodic solutions for the VCCD equation. Also by using a travelling wave transformation, we have reduced the VCCD equation to an ordinary differential equation, by the extended tanh function method we have been able to obtain many other new exact solitary and periodic-type solutions. Some remarks have been found on the obtained so-n lutions

Remark 1: The obtained Bäcklund transformation is more easy and simple in calculations than that obtained in [1] by using Painlevé-test. Additionally, the obtained solutions are also new and more general than solutions in Ref. [1] because all solutions in Ref. [1] depend on only one variable $\beta(t)$.

Remark 2: The combination between the two functions $\alpha(t)$ and $\beta(t)$ affects the propagation shape of the solitary wave solution. Moreover, the one-soliton solution $u_{1}$ recovers the single soliton solution obtained by Wazwaz in [5] for the CBS equation.

Remark 3: All solutions obtained in this paper have been satisfied by Mathematica program.

\section{References}

[1] Bansal A. and Gupta, R.K. (2012) Lie Point Symmetries and Similarity Solutions of the Time-Dependent Coefficients Calogero-Degasperis Equation. Physica Scripta, 86, 035005. http://dx.doi.org/10.1088/0031-8949/86/03/035005

[2] Wazwaz, A.M. (2008) New Solutions of Distinct Physical Structures to High-Dimensional Nonlinear Evolution Equations. Applied Mathematics and Computation, 196, 363-370. http://dx.doi.org/10.1016/j.amc.2007.06.002 
[3] Peng, Y. (2006) New Types of Localized Coherent Structures in the Bogoyavlenskii-Schiff Equation. International Journal of Theoretical Physics, 45, 1764-1768. http://dx.doi.org/10.1007/s10773-006-9139-7

[4] Bruzon, M.S., Gandarias, M.L., Muriel, C., Ramierez, J., Saez, S. and Romero, F.R. (2003) The Calogero-Bogoyavlenskii-Schff Equation in (2 + 1) Dimensions. Journal of Theoretical and Mathematical Physics, 137, 1367-1377.

[5] Wazwaz, A.M. (2008) Multiple-Soliton Solutions for the Calogero-Bogoyavlenskii-Schiff, Jimbo-Miwa and YTSF Equation. Applied Mathematics and Computation, 203, 592-597.

[6] Moatimid G.M., El-Shiekh R.M. and A.-G. A.A.H. Al-Nowehy (2013) Exact Solutions for Calogero-BogoyavlenskiiSchiff Equation Using Symmetry Method. Applied Mathematics and Computation, 220, 455-462.

[7] Fan, E. (2000) Two New Applications of the Homogeneous Balance Method. Physics Letter A, 265, 353-357. http://dx.doi.org/10.1016/S0375-9601(00)00010-4

[8] Fan, E. (2002) Auto-Bäcklund Transformation and Similarity Reductions for General Variable Coefficient KdV Equations. Physics Letter A, 294, 26-30.

[9] Moussa, M.H.M. and El Shikh, R.M. (2008) Auto-Bäcklund Transformation and Similarity Reductions to the Variable Coefficients Variant Boussinesq System. Physics Letter A, 372, 1429-1434.

[10] Moussa, M.H.M. and El Shikh, R.M. (2009) Two Applications of the Homogeneous Balance Method for Solving the Generalized Hirota-Satsuma Coupled KdV System with Variable Coefficients. International Journal of Nonlinear Science, 7, 29-38.

[11] Hang, Y. and Shang, Y.D. (2012) The Bäcklund Transformations and Abundant Exact Explicit Solutions for a General Nonintegrable Nonlinear Convection-Diffusion Equation. Abstract and Applied Analysis, 2012, 1-11. http://dx.doi.org/10.1155/2012/489043

[12] El Shiekh, R.M. and Al-Nowehy, A.-G. (2013) Integral Methods to Solve the Variable Coefficient Nonlinear Schrödinger Equation. Zeitschrift für Naturforschung, 68a, 255-260. http://dx.doi.org/10.5560/ZNA.2012-0108

[13] El Shiekh, R.M. (2015) Direct Similarity Reduction and New Exact Solutions for the Variable-Coefficient KadomtsevPetviashvili Equation. Zeitschrift für Naturforschung A, 70, 445-450. http://dx.doi.org/10.1515/zna-2015-0057

[14] El-Wakil, S.A., Abdou, M.A. and Hendi, A. (2008) New Periodic and Soliton Solutions of Nonlinear Evolution Equations. Applied Mathematics and Computation, 197, 497-506. http://dx.doi.org/10.1016/j.amc.2007.08.090

[15] Veksler, A. and Zarmi, Y. (2005) Wave Interactions and the Analysis of the Perturbed Burgers Equation. Physica D: Nonlinear Phenomena, 211, 57-73. http://dx.doi.org/10.1016/j.physd.2005.08.001

[16] Yu, X., Gao, Y.-T., Sun, Z.-Y. and Liu, Y. (2010) N-Soliton Solutions, Bäcklund Transformation and Lax Pair for a Generalized Variable-Coefficient Fifth-Order Korteweg-de Vries Equation. Physica Scripta, 81, 045402. http://dx.doi.org/10.1088/0031-8949/81/04/045402

[17] Jaradat, H.M., Al-Shara, S., Awawdeh, F. and Alquran, M. (2012) Variable Coefficient Equations of the KadomtsevPetviashvili Hierarchy: Multiple Soliton Solutions and Singular Multiple Soliton Solutions. Physica Scripta, 85, Article ID: 035001. http://dx.doi.org/10.1088/0031-8949/85/03/035001 